\title{
SOME INEQUALITIES FOR UNITARILY INVARIANT NORMS
}

\begin{abstract}
XINGKAI HU
Abstract. This paper aims to present some inequalities for unitarily invariant norms. In section 2, we give a refinement of the Cauchy-Schwarz inequality for matrices. In section 3, we obtain an improvement for the result of Bhatia and Kittaneh [Linear Algebra Appl. 308 (2000) 203-211]. In section 4, we establish an improved Heinz inequality for the Hilbert-Schmidt norm. Finally, we present an inequality involving positive definite matrix and Hilbert-Schmidt norm. Then we use it to discuss the conjecture on the Hilbert-Schmidt norm of matrices proposed by Sloane and Harwit and the conjecture is proved for some special matrices.
\end{abstract}

Mathematics subject classification (2010): 15A18; 15A42; 15A60.

Keywords and phrases: Unitarily invariant norms, positive semidefinite matrices, Cauchy-Schwarz inequality, convex function, Heinz inequality.

\section{REFERENCES}

[1] S. WANG, L. Zou, Y. JIANG, Some inequalities for unitarily invariant norms of matrices, J. Inequal. Appl. 2011, 2011:10.

[2] R. A. Horn, R. Mathias, Cauchy-Schwarz inequalities associated with positive semidefinite matrices, Linear Algebra Appl. 142 (1990) 63-82.

[3] R. A. Horn, R. MATHIAS, An analog of the Cauchy-Schwarz inequality for Hadamard products and unitarily invariant norms, SIAM J. Matrix Anal. Appl. 11 (1990) 481-498.

[4] R. BhATIA, C. DAVIS, A Cauchy-Schwarz inequality for operators with applications, Linear Algebra Appl. 223/224 (1995) 119-129.

[5] R. Bhatia, Matrix Analysis, Springer-Verlag, 1997.

[6] F. HIAI, X. ZHAN, Inequalities involving unitarily invariant norms and operator monotone functions, Linear Algebra Appl. 341 (2002) 151-169.

[7] R. Bhatia, F. Kittaneh, Notes on matrix arithmetic-geometric mean inequalities, Linear Algebra Appl. 308 (2000) 203-211.

[8] F. Kittaneh, Norm inequalities for fractional powers of positive operators, Lett. Math. Phys. 27 (1993) 279-285.

[9] R. Bhatia, C. DaVIS, More matrix forms of the arithmetic-geometric mean inequality, SIAM J. Matrix Anal. Appl. 14 (1993) 132-136.

[10] F. Kittaneh, Y. Manasrah, Improved Young and Heinz inequalities for matrices, J. Math. Anal. Appl. 361 (2010) 262-269.

[11] X. Zhan, Matrix theory, Beijing: Higher Education Press, 2008.

[12] N. J. A. Sloane, M. Harwit, Masks for Hadamard transform optics, and weighing designs, Appl. Optics. 15 (1976) 107-114.

[13] L. Zou, Y. Jiang, X. HU, A note on a conjecture on the Frobenius norm of matrices, Journal of Shandong University (Natural Science), 45 (4) (2010) 48-50.

[14] L. Zou, On a conjecture concerning the Frobenius norm of matrices, Linear Multilinear Algebra. 60 (2012) 27-31. 